\title{
BMJ open Expression of transforming growth factor $\beta$ receptor II in mesenchymal stem cells from systemic sclerosis patients
}

\author{
Valérie Vanneaux, ${ }^{1,2,3}$ Dominique Farge-Bancel, ${ }^{4,5}$ Séverine Lecourt, ${ }^{1,2,3}$ \\ Julie Baraut, ${ }^{5}$ Audrey Cras, ${ }^{1,2,3}$ Francette Jean-Louis, ${ }^{5}$ Cécilia Brun, ${ }^{5}$ \\ Franck Verrecchia, ${ }^{6}$ Jérôme Larghero, ${ }^{1,2,3}$ Laurence Michel $^{3,4,5}$
}

To cite: Vanneaux V, FargeBancel D, Lecourt S, et al. Expression of transforming growth factor $\beta$ receptor II in mesenchymal stem cells from systemic sclerosis patients. BMJ Open 2013;3:e001890. doi:10.1136/bmjopen-2012001890

\section{- Prepublication history for this paper are available online. To view these files please visit the journal online (http://dx.doi.org/10.1136/ bmjopen-2012-001890).}

Received 27 July 2012 Revised 18 September 2012 Accepted 28 September 2012

This final article is available for use under the terms of the Creative Commons Attribution Non-Commercial 2.0 Licence; see http://bmjopen.bmj.com

For numbered affiliations see end of article

\section{Correspondence to}

Dr Laurence Michel; laurence. michel@inserm.fr

\section{ABSTRACT}

Objectives: The present work aimed to evaluate the expression of transforming growth factor- $\beta$ (TGF- $\beta$ ) receptors on bone marrow-derived multipotent mesenchymal stromal cells (MSCs) in patients with systemic sclerosis (SSc) and the consequences of TGF- $\beta$ activation in these cells, since MSC have potential therapeutic interest for SSc patients and knowing that TGF- $\beta$ plays a critical role during the development of fibrosis in SSc.

Design: This is a prospective research study using MSC samples obtained from SSc patients and compared with MSC from healthy donors.

Setting: One medical hospital involving collaboration between an internal medicine department for initial patient recruitment, a clinical biotherapeutic unit for MSC preparation and an academic laboratory for research.

Participants: 9 patients with diffuse SSc for which bone marrow (BM) aspiration was prescribed by sternum aspiration before haematopoietic stem cell transplantation versus nine healthy donors for normal BM.

Primary and secondary outcome measures: TGF- $\beta$, TGF- $\beta$ receptor types I (TBRI) and II (TBRII) mRNA and protein expression were assessed by quantitative PCR and flow cytometry, respectively, in MSC from both SSc patients and healthy donors. MSC were exposed to TGF- $\beta$ and assessed for collagen $1 \alpha 2$ synthesis and Smad expression. As positive controls, primary cultures of dermal fibroblasts were also analysed.

Results: Compared with nine controls, MSC from nine SSc patients showed significant increase in mRNA levels $(p<0.002)$ and in membrane expression $(p<0.0001)$ of TBRII. In response to TGF- $\beta$ activation, a significant increase in collagen $1 \alpha$ synthesis $(p<0.05)$ and Smad-3 phosphorylation was upregulated in SSc MSC. Similar results were obtained on eight SSc-derived dermal fibroblasts compared to six healthy controls.

Conclusions: TBRII gene and protein expression defect in MSC derived from SSc patients may have pathological significance. These findings should be taken into account when considering the use of MSC-based therapies in an autologous setting.

\section{ARTICLE SUMMARY}

Article focus

- In systemic sclerosis (SSc), transforming growth factor- $\beta$ (TGF- $\beta$ ) seems pivotal since the extent of TGF- $\beta$ signalling activation in SSc skin fibroblasts correlated with disease severity and constitutes the best characterised therapeutic target in SSc fibrosis.

- Although multipotent progenitor mesenchymal stem cells (MSCs) from SSc patients exhibited similar differentiation potentials and immunosuppressive properties to that compared with their healthy counterparts, their response to TGF- $\beta$ has not been studied.

- We wished to determine if TGF- $\beta$ signalling in MSC was altered in SSc.

Key messages

- The results evidence an increase in TGF- $\beta$ receptor type II (TBRII) gene and protein expression in MSC derived from SSc patients as compared to MSC derived from healthy donors.

- Regarding that MSC may differentiate into tissue fibroblasts, these results suggest that the TBRII alteration observed in peripheral dermal fibroblasts from SSc patients might derive from a defect in their parental MSCs.

- The present results question the use of autologous MSC from SSc patients, alone or as adjuvant therapeutic product.

Strengths and limitations of this study

- Although limited by the small numbers of SSc patients, this is the first study revealing a defect in MSC derived from SSc patients and these data may have potent pathological significance in SSc pathophysiology.

- If these findings should be taken into account when discussing considering the use of MSC-based therapies in an autologous setting, functional experiments must be designed to validate the consequences of the defect of patients' MSC described in this in vitro study. 


\section{INTRODUCTION}

Systemic sclerosis (SSc) is a chronic systemic disease characterised by autoimmunity, vascular lesions and progressive fibrosis, with heterogeneous clinical presentation. ${ }^{1}$ As shown in vitro, SSc skin fibroblasts are characterised by abnormal growth and are responsible for abnormal extracellular matrix (ECM) accumulation. ${ }^{2}$ Multiple factors and signalling pathways are involved in the development or persistence of the SSc fibroblast phenotype. ${ }^{3}$ Although its exact relevance and interplay are still to be determined, transforming growth factor- $\beta$ (TGF- $\beta$ ), which is mainly produced by fibroblasts, as well as by $\mathrm{T}$ helper type 2 lymphocytes, is the major cytokine involved in collagen production leading to fibrosis. ${ }^{4}$ TGF- $\beta$ binds to transmembrane receptors that have intrinsic serine/threonine kinase activity. TGF- $\beta$ receptor type II (TBRII) links TGF- $\beta$, and the TBRI is then recruited into a heteromeric complex. TBRII transphosphorylates the glycine/serine rich domain of TBRI kinase. Following the phosphorylation of Smad-2 or Smad-3 by the activated TBRI, a heteromeric complex is formed with Smad-4, resulting in translocation of the complex into the nucleus. This complex acts directly as a transcription activator or indirectly regulates gene transcription by interacting with other transcription factors. TGF- $\beta$ receptors expression level is a critical mechanism for regulating the cellular response to cytokines. In particular, upregulation of TBR expression has been demonstrated in fibrotic diseases such as SSc, resulting in the deposition of ECM components. TGF- $\beta$ also stimulates the production of protease inhibitors, which prevent enzymatic breakdown of the ECM. We previously demonstrated that the extent of TGF- $\beta$ signalling activation in SSc skin fibroblasts correlated with disease severity. ${ }^{5}{ }^{6}$ Moreover, increased circulating TGF- $\beta$ levels have been observed in patients with $\mathrm{SSc}^{2}{ }^{2} 8$

Mesenchymal stem cells (MSCs) are multipotent progenitor cells, which display inhibitory function on T, B, natural killer and dendritic cells and represent promising

Table 1 Systemic sclerosis (SSc) patients' clinical characteristics: total or cutaneous biopsy site modified Rodnan Skin Score (mRSS)

\begin{tabular}{llll}
\hline $\begin{array}{l}\text { SSc } \\
\text { patients }\end{array}$ & $\begin{array}{l}\text { Age (years) at } \\
\text { bone marrow } \\
\text { aspiration }\end{array}$ & $\begin{array}{l}\text { Total } \\
\text { mRSS }\end{array}$ & $\begin{array}{l}\text { Cutaneous } \\
\text { mRSS }\end{array}$ \\
\hline 1 & 60 & 30 & 1 (Abdomen) \\
2 & 54 & 37 & 2 (Abdomen) \\
3 & 31 & 2 & 1 (Thigh) \\
4 & 37 & 25 & 1 (Thigh) \\
5 & 29 & 24 & 2 (Abdomen) \\
6 & 56 & 24 & 2 (Abdomen) \\
7 & 25 & 15 & 1 (Thigh) \\
8 & 25 & 16 & 2 (Thigh) \\
9 & 55 & 4 & 1 (Thigh) \\
Mean & 41 & 20 & \\
\hline
\end{tabular}

therapeutic tool. ${ }^{9-13}$ These immunosuppressive properties, in addition to their potential usefulness for tissue repair have led to use autologous MSC for treating graft versus host disease and several types of autoimmune diseases including SSc. ${ }^{14}$ We previously demonstrated that in vitro MSC from SSc patients exhibit similar differentiation potentials and immunosuppressive properties compared with their healthy counterparts. ${ }^{15}$ However, it is still unknown as to whether MSC from SSc patients displayed similar abnormalities as those observed SSc patient-derived fibroblasts, which are of particular interest since MSC may differentiate into fibroblasts. ${ }^{16}$ We therefore designed the present prospective research study to analyse TGF- $\beta$ signalling in MSC and in skin fibroblasts from SSc patients.

\section{MATERIALS AND METHODS}

\section{Cell cultures and characterisation}

Mesenchymal stem cells

Bone marrow (BM) samples were obtained by sternum aspiration in SSc patients $(\mathrm{n}=9)$, which characteristics are in table 1, prior to autologous haematopoietic stem cell transplantation for rapidly progressive disease. BM samples from healthy donors $(n=9)$ were obtained from filters used during BM processing for allogeneic transplantation. Approval from ethics committee from Hôpital Saint-Louis and signed consent were obtained for donors and patients.

BM-derived MSCs were isolated and expanded as previously described, ${ }^{15}$ in minimum essential medium- $\alpha$ (Invitrogen, Cergy Pontoise, France), supplemented with $10 \%$ foetal calf serum (HyClone, Logan, UK), L-glutamine (2 mM; Invitrogen), basic fibroblast growth factor $(1 \mathrm{ng} / \mathrm{ml}$; R\&D Systems, Lille, France) and antibiotic/antimycotic (Invitrogen).

Monoclonal antibodies conjugated with either fluorescein isothiocyanate or phycoerythrin and directed to CD34, CD45, CD73, CD29, CD90, CD105, CD44, TBRII or matched isotype control (Becton Dickinson, Le Pont de Claix, France, except TBRII, Sigma, St Louis, Missouri, USA) were used for immunophenotyping, according to the manufacturer's protocol. Data from patients and controls were acquired and analysed on a four parameter flow cytometer FACSCalibur using ProCellQuest software (BD Biosciences, San Jose, California, USA).

For activation experiments, MSC were cultured in the presence of 5 ng/ml TGF- $\beta 1$ (Peprotec, Lille, France).

\section{Fibroblasts}

Primary cultures of dermal fibroblasts derived from skin biopsies obtained during mammoplasty surgery (controls) or from SSc patients, at site with modified Rodnan Skin Score (mRSS) $\geq 2(n=9)$. Approval from ethics committee from Hôpital Saint-Louis and informed signed consent were obtained for both donors and patients. Cells were cultured in Dulbecco's Modified Eagle Medium containing $10 \%$ fetal bovine serum, $100 \mathrm{U} / \mathrm{ml}$ penicillin and $100 \mathrm{mg} / \mathrm{ml}$ streptomycin in a humidified 
incubator at $37^{\circ} \mathrm{C}$ with $5 \% \mathrm{CO}_{2}$ atmosphere. Fibroblasts were used between the third and fifth passage.

\section{RNA preparation and real-time PCR}

Reverse transcription-PCR analysis

Total RNA was extracted using an RNeasy mini-kit (Qiagen, Hiden, Germany), according to the manufacturer's instructions. DNase I treatment (25 units, $15 \mathrm{~min}$ ) of total RNA was performed to eliminate genomic contamination of the RNA samples. One microgram of total RNA was used for first strand cDNA synthesis using a RT-for-PCR kit (Invitrogen, Carlsbad, California, USA). Real-time PCR was performed with an ABI PRISM 7300 instrument (Applied Biosystems, Foster City, California, USA) using SYBRGreen PCR Core Reagents (Applied Biosystems). Gene expression was corrected against GAPDH and GUS mRNA level in each sample. The sequences of the forward and reverse primers for TGF- $\beta 1$, TBRI, TBRII, Smad-2, Smad-3, Smad-4, Smad-7, collagen(col)1 $\alpha 2$, GAPDH and GUS mRNA are available under request.

\section{ELISA}

TGF- $\beta 1$, metalloproteinase 2 and 9 levels in MSC culture supernatants were quantified by Quantikine ELISA according to the manufacturer's instruction (R\&D Systems).

\section{Statistical analyses}

Statistical significance for all experiments was assessed using the two-sided Student's t test. Differences were considered as significant at $\mathrm{p}<0.05$.

\section{RESULTS}

Increased expression of TBRII in MSC from SSc patients All SSc MSC highly expressed CD29, CD73, CD90, CD105 and adhesion molecules (CD44). The absence of contaminating haematopoietic stem cells was confirmed by the negative expression of CD34 and CD45 antigens. No phenotypic differences were observed between BM-MSCs from SSc patients and controls.

As compared with healthy donors, SSc MSC displayed significantly higher expression of TBRII mRNA, as measured by qRT-PCR $(p<0.002$; figure $1 A)$ and the percentage of TBRII receptors at the cell membrane was significantly increased $(\mathrm{p}<0.01)$ as the index of TBRII expression (TBRII\% $\times$ mean fluorescence intensity) $(\mathrm{p}<0.0001)$, as shown in figure $1 \mathrm{~B}$.

No significant difference in TBRI mRNA levels was observed between controls and SSc MSC, nor in the expression of Smad-2, Smad-3, Smad-4 and Smad-7 mRNA (figure 1A).

Although TGF- $\beta 1$ mRNA levels were higher in MSC from SSc patients than in controls (figure 1A), the increase was not significant. The levels of metalloproteinase 2,9 and TGF- $\beta 1$ in culture supernatants did not differ significantly between donors and SSc MSC (data not shown).

TGF- $\beta$-induced Smad pathway and collagen $1 \alpha 2$ synthesis are altered in MSC from SSc patients

In order to determine whether the increased TBRII expression was linked to Smad-pathway signalling, MSC were cultured in the presence of TGF- $\beta$. Target gene expression, including collagen $1 \alpha 2$, and Smad-3 phosphorylation were assessed. In response to TGF- $\beta$ activation, MSC from SSc patients expressed significantly more coll $\alpha 2$ mRNA than MSC from healthy donors during the first $12 \mathrm{~h}(\mathrm{p}<0.05)$ as shown in figure 2A. In addition, mRNA synthesis of plasminogen activator inhibitor (PAI-1) was increased by TGF- $\beta$ activation in MSC from SSc patients as compared with healthy MSC (data not shown).

By western blot analysis, a 10-fold increase in Smad-3 phosphorylation level was observed in MSC from SSc patients compared with healthy donors (figure 2B).

In accordance with data obtained on MSC, a significant increase in TBRII and coll $\alpha 2$ mRNA expression was also detected in dermal fibroblasts from SSc patients $(n=8)$ as compared with fibroblasts from normal skin $(\mathrm{n}=6$; figure $2 \mathrm{C})$.

We previously reported that the increase of Smad-3 phosphorylation and of col1 $\alpha 2$ mRNA levels parallels the mRSS values and therefore relates to the histological grade of fibrosis. In the present study, MSC were obtained from patients with a mean mRSS Rodnan score of $19.7 \pm 3.8(n=9$; table 1$)$. This mean value revealed that cutaneous fibrosis was quite intense in the studied patients.

\section{DISCUSSION}

Our present analysis of TGF- $\beta$ signalling in MSC and in skin fibroblasts from SSc patients allowed us to demonstrate a significantly increased expression of TBRII in both MSC and skin fibroblasts from SSc patients that lead to alteration of the TGF- $\beta$-induced Smad pathway and collagen $1 \alpha 2$ synthesis in these cells. These data are in agreement with our previous results showing that TGF- $\beta$-dependent Smad pathway was increased in dermal SSc fibroblasts, ${ }^{6}$ and with previous reports showing that mRNA steady state levels of TGF- $\beta$ receptors, coll $\alpha 2$ and PAI-1 were increased in SSc fibroblasts as compared to their normal counterparts. ${ }^{3} 1718$

As TGF- $\beta$ receptors were shown to be up-regulated by several growth factors including TGF- $\beta$ itself, ${ }^{19}$ the overexpression of TBRII observed in MSCs from SSc patients as well as in dermal SSc fibroblasts could be due to exposure to their autocrine TGF- $\beta$ synthesis. Enhanced expression of TGF- $\beta$ receptors might favour SSc fibrosis since the overexpression of TGF- $\beta$ receptors was shown to induce collagen transcription in cultured dermal fibroblasts, ${ }^{18}$ and of dominant negative TBRII to inhibit ECM production in SSc fibroblasts. ${ }^{20}$ 


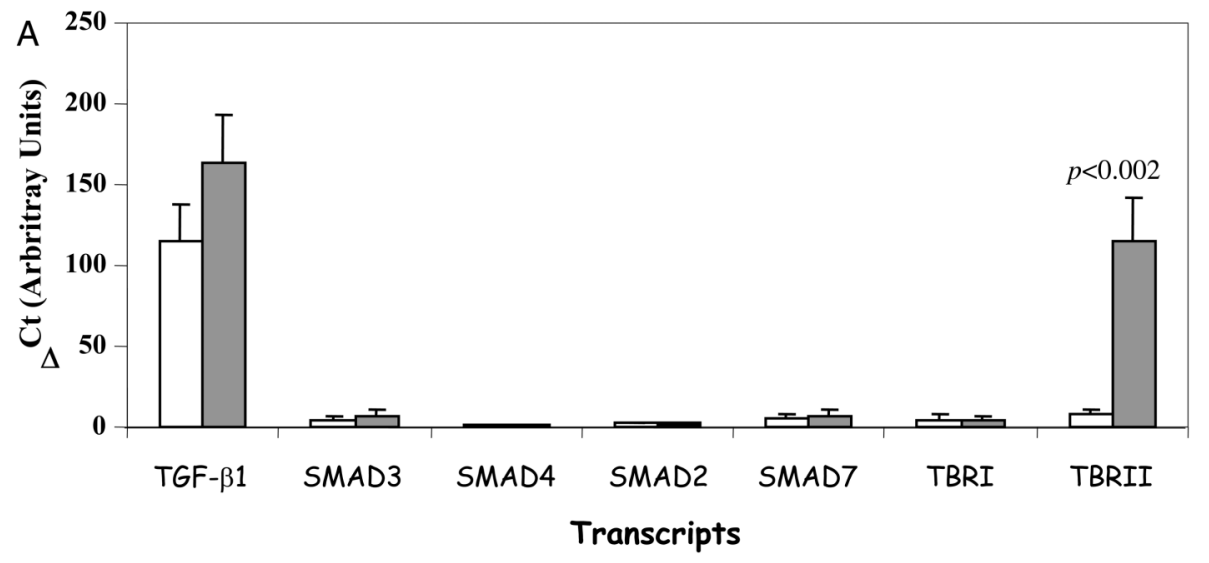

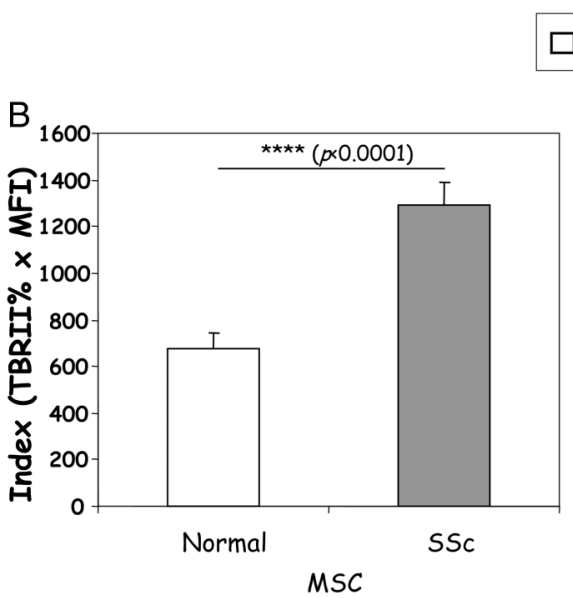

Mean Index $( \pm S D)=$ TBRII $\% \times$ MFI (Mean Fluorescence Intensity) ( $n=9$ Normal Donors, Normal) ( $n=9$ SSc patients, SSc).
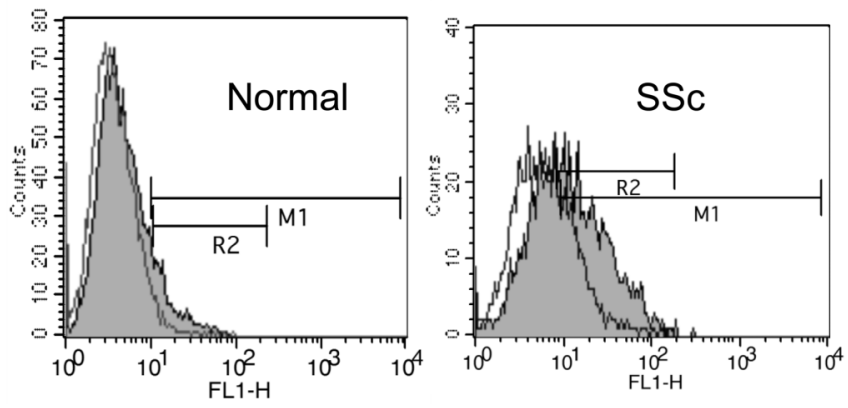

FACS analysis of TBRII Membrane Expression in MSC
from one representative donor (Normal)
and one representative SSC patient

Figure 1 Basal mRNA and TBRII membrane expression in MSC. MSC were derived from bone marrow aspirates of normal donors (normal MSC) and SSc patients (SSc MSC). (A) Basal mRNA levels of TBRII, TBRI, TGF- $\beta$, Smad-2, Smad-3, Smad-4 and Smad-7 were measured by real-time PCR in normal MSC $(n=6)$ and SSc MSC $(n=9)$. (B) TBRII membrane expression was quantified by mean fluorescence intensity (MFI) and percentage of positive cells (\%) using flow cytometry analysis. An index of membrane expression (TBRII\% xMFI) was calculated and expressed as mean \pm SD for normal MSC $(n=9)$ and SSc MSC ( $n=9$, left panel). TBRII expression on MSC from one representative normal donor (medium panel) and one representative SSc patient (right panel) is shown in grey, with overlaying of respective isotype controls. MSC, mesenchymal stromal cell; SSc, systemic sclerosis; TBRII, transforming growth factor- $\beta$ receptor type II.

In accordance with the recent demonstration that MSC could be differentiated into fibroblasts, ${ }^{16}$ our results suggest that the TBRII alteration observed in peripheral dermal fibroblasts from SSc patients might derive from a defect in their parental mesenchymal stem cells. It can be suggested that the TGF- $\beta$ hypersensitivity of MSC from SSc patients, as shown by the increase in coll $\alpha 2$ mRNA expression in response to the mediator, might favour their own differentiation into fibroblasts and/or organ fibrosis. The relation we observed between the increase of TGF- $\beta$-signalling pathway in both MSC and fibroblasts with the fibrosis histological grade, as measured by mRSS score, favours the link between central mesenchymal stem cell defect and peripheral fibrosis in SSc patients. However, further studies with larger numbers of SSc patients will be helpful for refined analysis of TBR expression in SSc MSC and SSc fibroblasts and better understanding of their potent impact on cell lineage.

The present results question the use of autologous MSC from SSc patients, alone or as adjuvant therapeutic product. While in vitro SSc-MSC immunomodulatory properties have been found to be similar to that of healthy donors, ${ }^{15}$ other studies revealed that SSc-MSC displayed impaired endothelial cell differentiation capacities, ${ }^{21}$ and overexpressed and secreted proangiogenic factors such as VEGF and SDF-1. ${ }^{22}$ These points should be carefully considered when choosing the source of MSC, allogeneic or autologous, for clinical use in SSc patients. 
A Coll $1 \alpha$
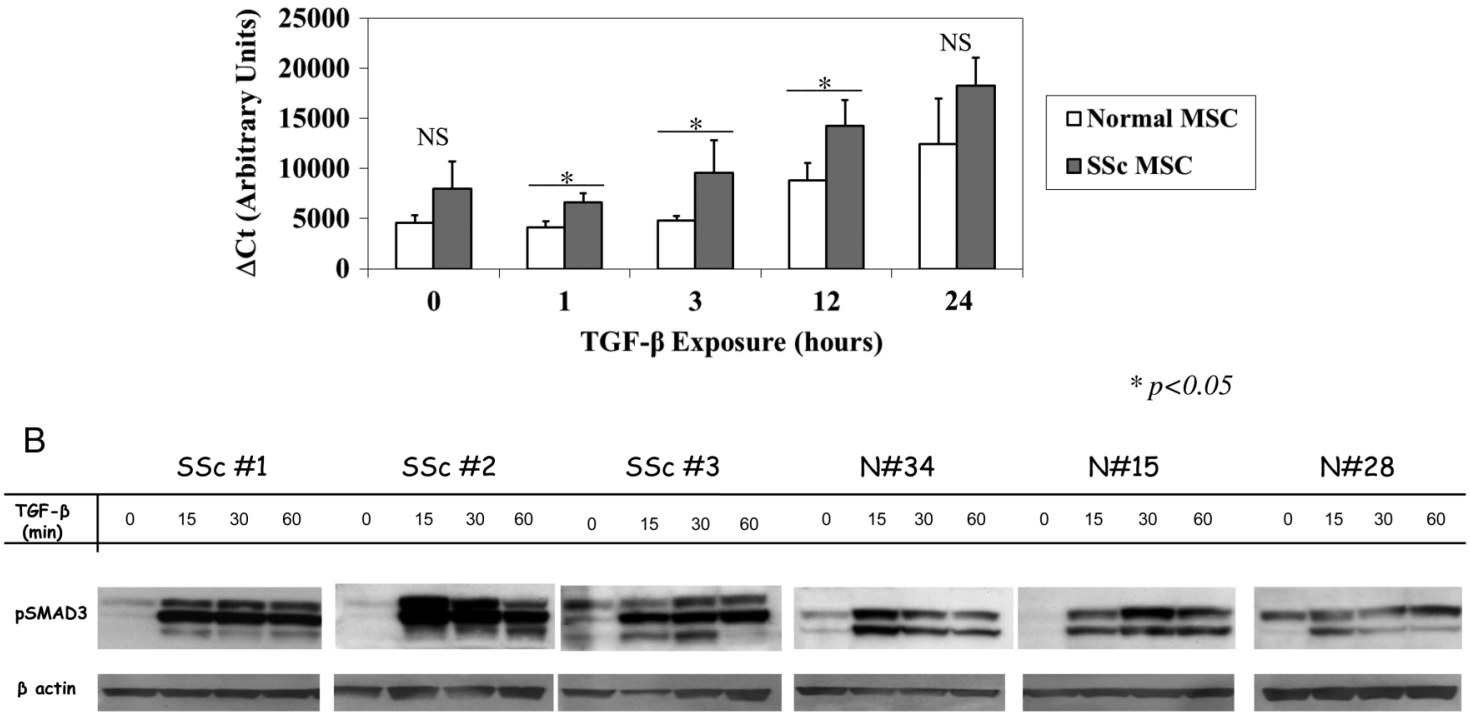

$50 \mu \mathrm{g}$ total extracts

C

TBRII mRNA levels in fibroblasts

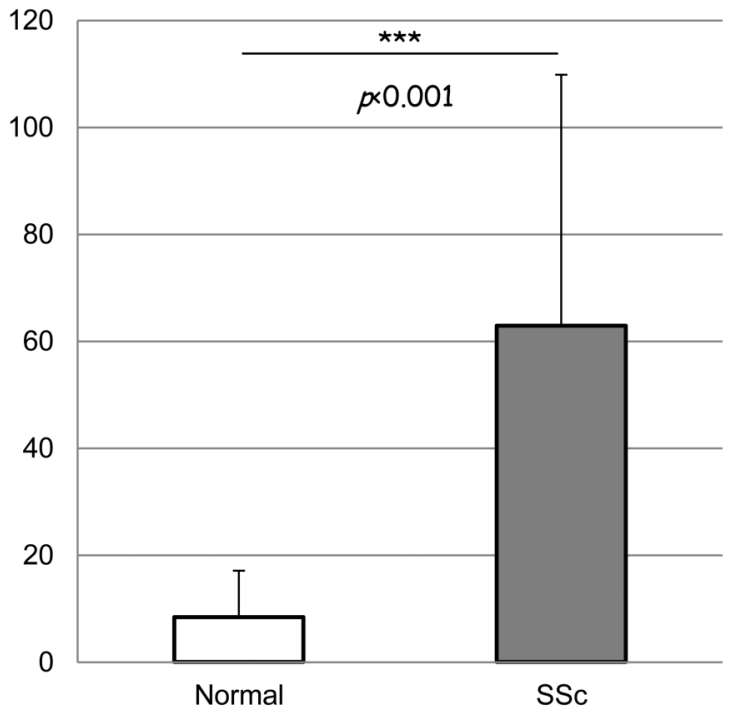

Coll1 $\alpha 2$ mRNA levels in fibroblasts

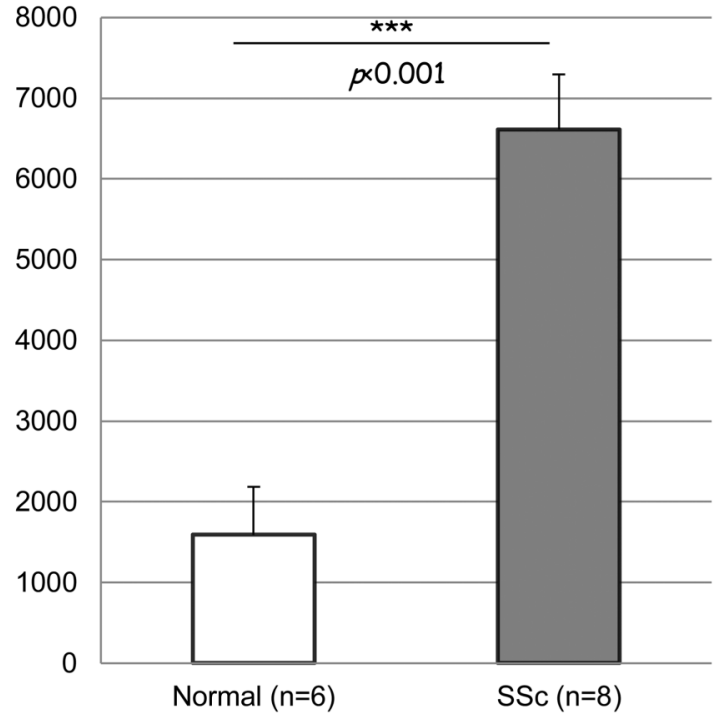

Figure 2 Activation of the TGF- $\beta$-induced Smad pathway and increased synthesis of collagen $1 \alpha$ in MSC from SSc patients. (A) Normal $(n=6)$ and SSc MSC ( $n=6)$ were cultured in the presence of TGF- $\beta$ during 1, 3, 12 and $24 \mathrm{~h}$. Collagen $1 \alpha 2 \mathrm{mRNA}$ (Coll1 $\alpha 2$ ) was measured by real-time PCR. ${ }^{*} \mathrm{p}<0.05$. (B) SSc MSC (left panels) and control MSC (right panels) were cultured with $5 \mathrm{ng} / \mathrm{ml} \mathrm{TGF-} \beta$ during 15, 30 and $60 \mathrm{~min}$. Phosphorylated Smad-3 and respective $\beta$-actin protein expressions were analysed by western blotting. (C) Primary cultures of dermal fibroblasts were derived from skin of healthy donors $(n=6)$ or SSc patients $(n=8)$. Basal TBRII (left) and coll1 $\alpha 2$ (right) mRNA levels were measured by real-time PCR. MSC, mesenchymal stromal cell; SSc, systemic sclerosis; TGF- $\beta$, transforming growth factor- $\beta$.

\section{CONCLUSION}

Our study reveals that the expression of TBRII is significantly increased in SSc MSC, with a consequent defect in the production of collagen type $1 \alpha 2$ in response to TGF- $\beta$ activation. This defect in the MSC upstream may have a pathological significance in SSc. The present results question the use of autologous MSC from SSc patients, alone or as adjuvant therapeutic product.
Author affiliations

${ }^{1}$ Unité de Thérapie Cellulaire et Centre d'Investigation Clinique en Biothérapies CIC-BT501, Assistance Publique-Hôpitaux de Paris, Hôpital Saint Louis, Paris, France

${ }^{2}$ INSERM UMR940, Institut Universitaire d'Hématologie, Paris, France

${ }^{3}$ Université Paris Diderot, Sorbonne Paris Cité, Paris, France

${ }^{4}$ Unité de Médecine Interne et Pathologie Vasculaire, Assistance Publique-Hôpitaux de Paris, Hôpital Saint Louis, Paris, France

${ }^{5}$ INSERM UMR976 Skin Research Center and Université Paris Diderot, Sorbonne Paris Cité, Hôpital Saint-Louis, Paris, France

${ }^{6}$ INSERM UMR957 and Université Nantes Atlantique, Nantes, France 
Acknowledgements We acknowledge the 'Association des Sclérodermiques de France' (ASF) and the 'Groupe Français de Recherche sur la Sclérodermie' (GFRS).

Contributors VV, SL, JB, AC, FJ-L and CB participated in sample handling and laboratory work. VV, DF-B, FV, JL and LM participated in data analyses. VV and LM participated in manuscript writing. Approval of final manuscript was done by VV, DF-B, SL, JB, AC, FJ-L, CB, FV, JL and LM.

Funding This work was supported by grants from the "Groupe Français de Recherche sur la Sclérodermie" (GFRS).

Competing interests None.

Patient consent Obtained.

Ethics approval Ethics committee of Saint-Louis Hospital.

Provenance and peer review Not commissioned; externally peer reviewed.

Data sharing statement No additional data are available.

\section{REFERENCES}

1. Gabrielli A, Avvedimento EV, Krieg T. Scleroderma. N Engl J Med 2009;360:1989-2003.

2. Verrecchia F, Mauviel A, Farge D. Transforming growth factor-beta signaling through the Smad proteins: role in systemic sclerosis. Autoimmun Rev 2006;5:563-9.

3. Abraham DJ, Varga J. Scleroderma: from cell and molecular mechanisms to disease models. Trends Immunol 2005;26:587-95.

4. Massague J. How cells read TGF-beta signals. Nat Rev Mol Cell Biol 2000;1:169-78.

5. Ihn H, Yamane K, Kubo M, et al. Blockade of endogenous transforming growth factor beta signaling prevents up-regulated collagen synthesis in scleroderma fibroblasts: association with increased expression of transforming growth factor beta receptors. Arthritis Rheum 2001:44:474-80.

6. Verrecchia F, Laboureau J, Verola O, et al. Skin involvement in scleroderma-where histological and clinical scores meet. Rheumatology (Oxford) 2007;46:833-41.

7. Denton CP, Abraham DJ. Transforming growth factor-beta and connective tissue growth factor: key cytokines in scleroderma pathogenesis. Curr Opin Rheumatol 2001:13:505-11.

8. Ihn H. Autocrine TGF-beta signaling in the pathogenesis of systemic sclerosis. J Dermatol Sci 2008:49:103-13.
9. Pittenger MF, Mackay AM, Beck SC, et al. Multilineage potential of adult human mesenchymal stem cells. Science 1999;284: 143-7.

10. Corcione A, Benvenuto F, Ferretti E, et al. Human mesenchymal stem cells modulate B-cell functions. Blood 2006;107:367-72.

11. Spaggiari GM, Capobianco A, Becchetti S, et al. Mesenchymal stem cell-natural killer cell interactions: evidence that activated NK cells are capable of killing MSCs, whereas MSCs can inhibit IL-2-induced NK-cell proliferation. Blood 2006;107:1484-90.

12. Dazzi F, Ramasamy R, Glennie S, et al. The role of mesenchymal stem cells in haemopoiesis. Blood Rev 2006;20:161-71.

13. Pittenger MF. Mesenchymal stem cells from adult bone marrow. Methods Mol Biol 2008;449:27-44.

14. Tyndall A, Uccelli A. Multipotent mesenchymal stromal cells for autoimmune diseases: teaching new dogs old tricks. Bone Marrow Transplant 2009:43:821-8.

15. Larghero J, Farge D, Braccini A, et al. Phenotypical and functional characteristics of in vitro expanded bone marrow mesenchymal stem cells from patients with systemic sclerosis. Ann Rheum Dis 2008;67:443-9.

16. Lee $\mathrm{CH}$, Shah $\mathrm{B}$, Moioli EK, et al. CTGF directs fibroblast differentiation from human mesenchymal stem/stromal cells and defines connective tissue healing in a rodent injury model. $J$ Clin Invest 2010;120:3340-9.

17. Verrecchia F, Mauviel A. Transforming growth factor-beta and fibrosis. World J Gastroenterol 2007;13:3056-62.

18. Kawakami T, Ihn $\mathrm{H}$, Xu W, et al. Increased expression of TGF-beta receptors by scleroderma fibroblasts: evidence for contribution of autocrine TGF-beta signaling to scleroderma phenotype. J Invest Dermatol 1998;110:47-51.

19. Yamane $\mathrm{K}$, Ihn H, Asano $\mathrm{Y}$, et al. Antagonistic effects of TNF- $\alpha$ on TGF- $\beta$ signaling through down-regulation of TGF- $\beta$ receptor type II in human dermal fibroblasts. J Immunol 2003;171:3855-62.

20. Trojanowska M, Smith E, Dong JY, et al. Overexpression of a kinase-deficient TGF $\beta$ receptor II via an adenoviral vector potently inhibits extracellular matrix production in scleroderma and normal dermal fibroblasts (abstract 795). Arthritis Rheumatism 1999;42: S203.

21. Cipriani P, Guiducci S, Miniati I, et al. Impairment of endothelial cell differentiation from bone marrow-derived mesenchymal stem cells: new insight into the pathogenesis of systemic sclerosis. Arthritis Rheum 2007;56:1994-2004.

22. Guiducci S, Manetti M, Romano E, et al. Bone marrow-derived mesenchymal stem cells from early diffuse systemic sclerosis exhibit a paracrine machinery and stimulate angiogenesis in vitro. Ann Rheum Dis 2011;70:2011-21. 